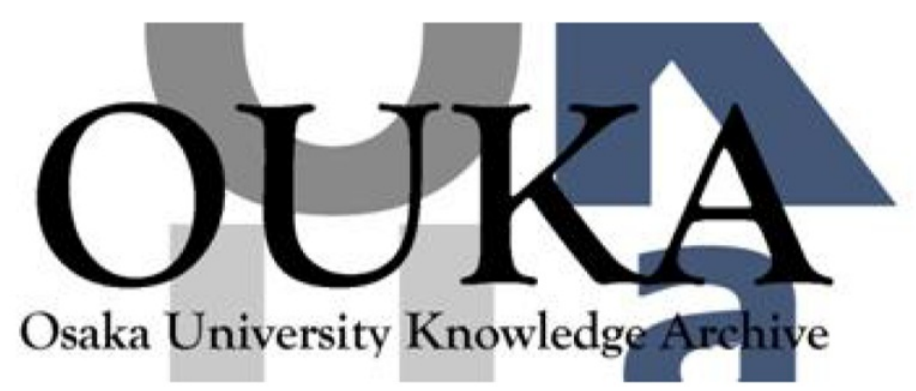

\begin{tabular}{|c|l|}
\hline Title & $\begin{array}{l}\text { Calibration of imaging plate for high energy } \\
\text { electron spectrometer }\end{array}$ \\
\hline Author(s) & $\begin{array}{l}\text { Tanaka, Kazuo A.; Yabuuchi, Toshinori; Sato, } \\
\text { Takashi et al. }\end{array}$ \\
\hline Citation & $\begin{array}{l}\text { Review of Scientific Instruments. 76(1) } \\
\text { p. } 013507\end{array}$ \\
\hline Issue Date & $2005-01$ \\
\hline oaire:version & VoR \\
\hline URL & https://hdl. handle. net/11094/3116 \\
\hline rights & \\
\hline Note & \\
\hline
\end{tabular}

Osaka University Knowledge Archive : OUKA

https://ir. Library. osaka-u. ac. jp/

Osaka University 


\title{
Calibration of imaging plate for high energy electron spectrometer
}

\author{
Kazuo A. Tanaka ${ }^{\text {a) }}$ \\ Faculty of Engineering and Institute of Laser Engineering, Osaka University, Yamada-oka, 2-6, Suita, \\ Osaka 565-0871, Japan \\ Toshinori Yabuuchi, Takashi Sato, Ryosuke Kodama, and Yoneyoshi Kitagawa \\ Institute of Laser Engineering, Osaka University, Yamada-oka, 2-6, Suita, Osaka 565-0871, Japan \\ Teruyoshi Takahashi \\ Department of Electrical Engineering, Kinki University, Higashi-Osaka, Osaka 577-8502, Japan \\ Toshiji Ikeda and Yoshihide Honda \\ The Institute of Scientific and Industrial Research, Osaka University, Ibaraki, Osaka 567-0047, Japan \\ Shuuichi Okuda \\ Research Institute for Advanced Science and Technology, Osaka Prefecture University, Sakai, \\ Osaka 599-8531, Japan
}

(Received 20 April 2004; accepted 27 September 2004; published online 22 December 2004)

\begin{abstract}
A high energy electron spectrometer has been designed and tested using imaging plate (IP). The measurable energy range extends from 1 to $100 \mathrm{MeV}$ or even higher. The IP response in this energy range is calibrated using electrons from L-band and S-band LINAC accelerator at energies $11.5,30$, and $100 \mathrm{MeV}$. The calibration has been extended to $0.2 \mathrm{MeV}$ using an existing data and Monte Carlo simulation Electron Gamma Shower code. The calibration results cover the energy from 0.2 to $100 \mathrm{MeV}$ and show almost a constant sensitivity for electrons over $1 \mathrm{MeV}$ energy. The temperature fading of the IP shows a $40 \%$ reduction after $80 \mathrm{~min}$ of the data taken at $22.5^{\circ} \mathrm{C}$. Since the fading is not significant after this time we set the waiting time to be $80 \mathrm{~min}$. The oblique incidence effect has been studied to show that there is a $1 / \cos \theta$ relation when the incidence angle is $\theta$. (C) 2005 American Institute of Physics. [DOI: 10.1063/1.1824371]
\end{abstract}

\section{INTRODUCTION}

An electron spectrometer is designed and tested to measure high-energy electrons generated from ultraintense laser plasma interactions. The electron spectrometer is exposed to a very strong electromagnetic pulse (EMP) noise originating from the ultraintense laser irradiation onto a target. In order to construct a detector not affected by EMP noise, we have employed imaging plate (IP). ${ }^{1,2}$ IP which makes use of photo-stimulated luminescence (PSL) effect $^{3}$ is a timeintegrated type detector for radiation such as x ray, electron, positron, and ion. Spatially distributed intensity information can be stored on IP and can be read out with a commercial reader. ${ }^{4}$ The energy of electrons generated by the laser irradiation may range from less than $1 \mathrm{MeV}$ to over $100 \mathrm{MeV}$. We have performed the calibration of IP for electron energy up to $100 \mathrm{MeV}$ in order to evaluate the electron numbers for PSL values, which is the signal intensity obtained by the readout system.

\section{ELECTRON SPECTROMETER WITH IP}

When an ultraintense (UI) laser pulse (focused intensity $>10^{18} \mathrm{~W} / \mathrm{cm}^{2}$ ) irradiates solid, plasma, or gas targets, highenergy electrons are generated. These electrons are produced

\footnotetext{
${ }^{\text {a) }}$ Author to whom correspondence should be addressed; electronic mail: katanaka@ile.osaka-u.ac.jp
}

through $\mathbf{J} \times \mathbf{B}$ acceleration, vacuum heating, etc., ${ }^{5,6}$ with energies up to hundreds of mega-electron-volts. These electrons can be utilized for various applications such as fast ignition in inertial fusion energy. ${ }^{7,8}$ We use IP as a detector of these energetic electrons. With the use of the electron spectrometers (ESM) it is possible to measure the details of electron spectrum. The measurement can be used to study the characteristics such as electron angular distribution, target material dependence, and laser parameter dependence. We have developed an ESM using IP as a detector. Compared to other conventional detectors the advantages of IP are a large dynamic range $\left(\sim 10^{5}\right)$ and a high sensitivity $(\sim 100$ times higher than a normal $\mathrm{x}$ ray film). ${ }^{1,2}$ IP is not affected by a strong EMP noise. Detectors with electrical circuits could be paralyzed frequently by a strong EMP noise or the signals

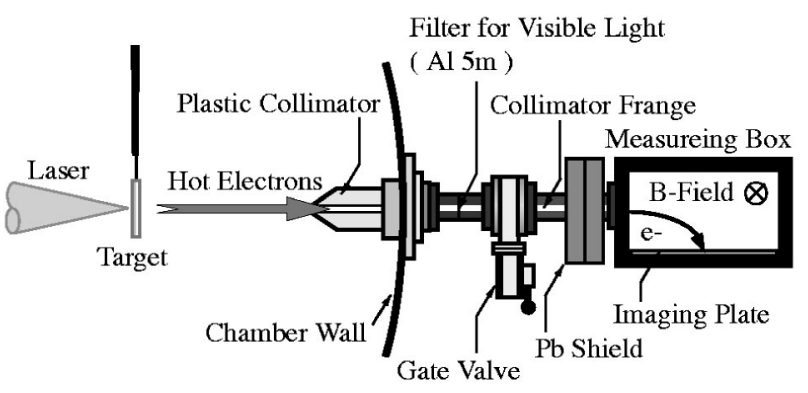

FIG. 1. Schematic of ESM for measuring energetic electrons generated by ultraintense laser-plasma interactions. 


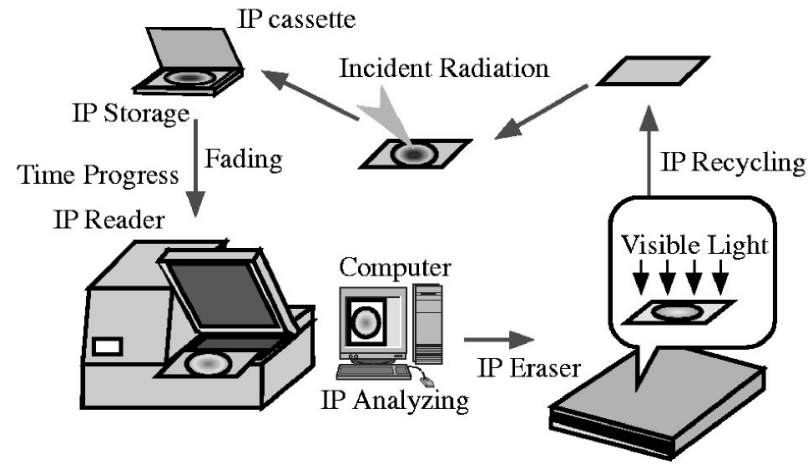

FIG. 2. Flow of IP data reduction.

could be overwhelmed by the noise. IP is free from such difficulties though the EMP noise may contribute to background noise. The background EMP noise could be reduced by building up proper noise blocks.

The layout of our ESM is shown in Fig. 1. The ESM can be attached on a vacuum chamber ( $2 \mathrm{~m}$ diameter) to detect the electrons through a collimating hole which size is $5 \mathrm{~mm}$ in diameter. The high energy electrons generated in the laser-plasma interactions are emitted from the target and pass though the collimator. The collimated hot electrons enter the magnetic field region. Electrons are dispersed by a permanent magnet (up to $4.5 \mathrm{kG}$ ) of different energy and are incident on IP at different location. The location of each electron is determined by a Larmor radius on which depends magnetic field and electron energy shown as

$$
R=\frac{m_{0} c}{e B} \sqrt{\left(\frac{\varepsilon+m_{0} c^{2}}{m_{0} c^{2}}\right)^{2}-1},
$$

where $R$ is the relativistic larmor radius for fast electrons with energy $\varepsilon$ in the magnetic field $B$.

Electrons with energies up to a maximum of $200 \mathrm{MeV}$ can be detected with the ESM. The resolution of the electron energy spectrum is $20 \mathrm{keV}$ at the lowest end and $400 \mathrm{keV}$ at the highest when a $200 \times 200 \mu \mathrm{m}$ square is set for signal read out on IP. The noise level which comes from the $\gamma$ rays is very high in ultraintense laser experiments. IP is placed along the line of the emission of the $\gamma$-ray flux to reduce the solid angle at IP to nearly zero for $\gamma$ rays. If enough thick plastic and lead shield can be set in the front of ESM and the noise level is quite small, then IP can be placed at the right angle to noise source in order to maximize the energy resolution. Also the energy resolution becomes better when the

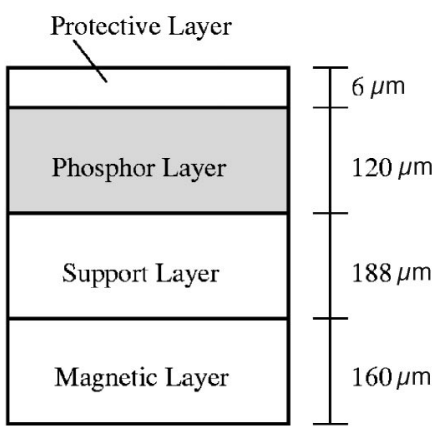

FIG. 3. Configuration of BAS-SR2025.

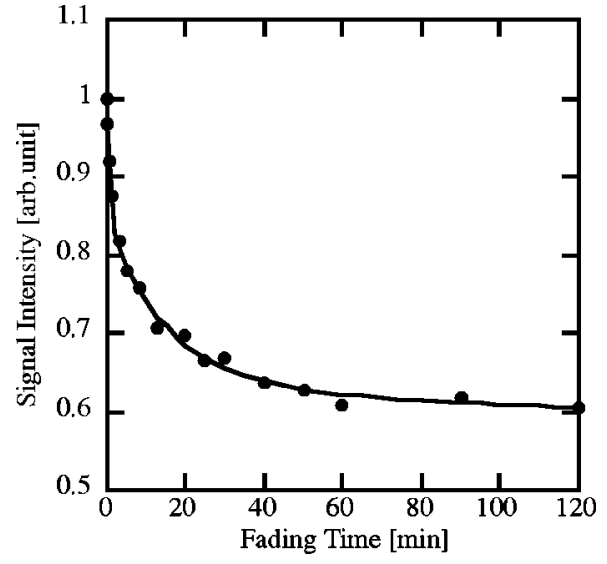

FIG. 4. Fading curve.

diameter of the collimator is reduced in the case in which the high energy electron number is very large to obtain signal with IP.

\section{IP CHARACTERIZATION}

IP has to be calibrated so that a given PSL value should correspond to the absolute number of electrons at each electron energy, when a spatially distributed electron spectrum is recorded on IP. In order to calibrate IP for a given electron energy up to $100 \mathrm{MeV}$ electrons of energies from 10 to $30 \mathrm{MeV}$ at the L-band LINAC and those from 30 to $100 \mathrm{MeV}$ at the S-band LINAC machines are used at the Institute of Scientific and Industrial Research, Osaka University.

\section{A. Fading of IP data}

IP can be processed as shown in Fig. 2. An IP containing electron data can be read out with a commercial reader. The reader can be operated with a PC and the read out data can be stored in the PC. The data in the IP can be erased with a visible light. Once the data are recorded on an IP, one needs to consider the fading effect of IP. The fading is the phenomena that the density of trapped electrons in the conduction band decreases in time. ${ }^{9}$ We should take into account this fading when the data read out is performed since there is always some time spent after the data acquisition. The fading depends on the type of IP and surrounding temperature. We have measured the fading in the typical experimental environment.

The IP used is BAS-SR2025 (Fuji Film) and the reader is BAS-1800. Shown in Fig. 3 is the layer structure of BASSR2025. The top layer is protective plastic layer of $6 \mu \mathrm{m}$.

TABLE I. Setting parameters of LINAC.

\begin{tabular}{lcc}
\hline \hline & L-band & S-band \\
\hline Electron energy (MeV) & $11.5,30$ & 100 \\
Charge (nC/shot) & 0.05 & 2.5 \\
Pulse duration (ns) & 5 & 5 \\
Output mode & Multibunch mode & Multibunch mode \\
\hline \hline
\end{tabular}




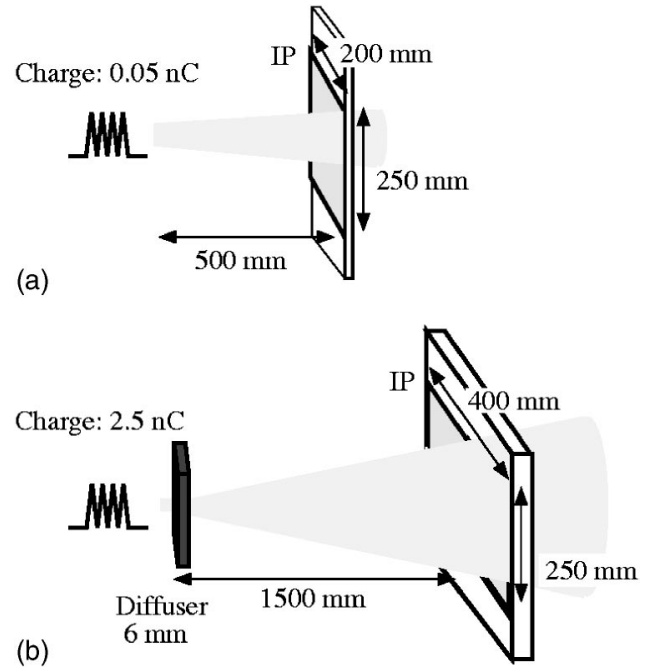

FIG. 5. The layout of the calibration experiments for (a) $11.5,30 \mathrm{MeV}$ (L-band) and (b) $100 \mathrm{MeV}$ (S-band) electrons.

The next is the phosphor layer of $120 \mu \mathrm{m}$ followed by support plastic and magnetic layers. The magnetic layer is used to stick the IP on the reader carrier.

We have exposed the IP to a $\beta$ ray source $\left({ }^{147} \mathrm{Pm}\right)$ for a minute at $22.5{ }^{\circ} \mathrm{C}$. We measured the fading at $22.5^{\circ} \mathrm{C}$. As shown in Fig. 4, the fading is sharp for the first $60 \mathrm{~min}$ and then becomes slow. The relative signal intensity at $t$ min after irradiation can be fitted to an empirical equation given in Eq. (2). This formula, $\exp (-\ln 2 \times t / T)$, is used in Ref. 10 for representing the relative intensity at $t$ min. The line in Fig. 4 is drawn using Eq. (2). Here $t$ is in minutes,

$$
\begin{aligned}
f(t)= & 0.16 \times \exp (-\ln 2 \times t / 0.56)+0.21 \times \exp (-\ln 2 \\
& \times t / 11)+0.63 \times \exp (-\ln 2 \times t / 1991) .
\end{aligned}
$$

Considering the fading characteristics, the recommended waiting time is longer than $60 \mathrm{~min}$. In our experiments, the IP was read out at $80 \mathrm{~min}$ after the data recording.

\section{B. Calibration of IP}

Electrons from a LINAC are used to irradiate IP at 11.5 and $30 \mathrm{MeV}$ from L-band and at $100 \mathrm{MeV}$ from S-band. The irradiation conditions are summarized in Table I. The L-band

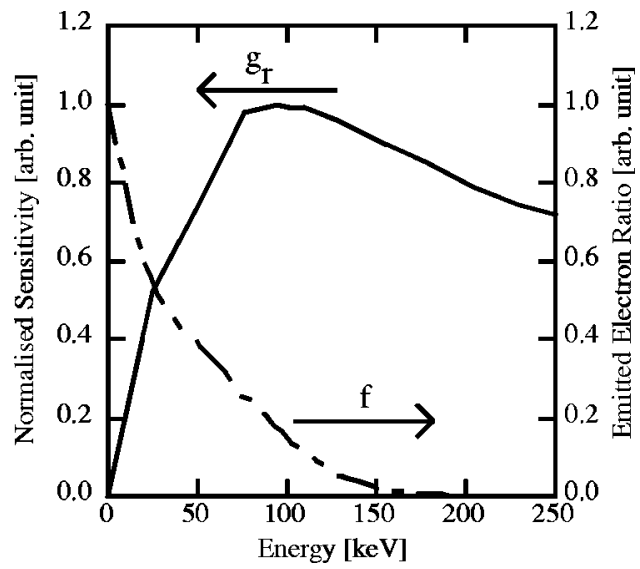

FIG. 6. Normalized sensitivity curve is $g(r)$ and energy spectrum of ${ }^{147} \mathrm{Pm}$ is $f(\varepsilon)$.

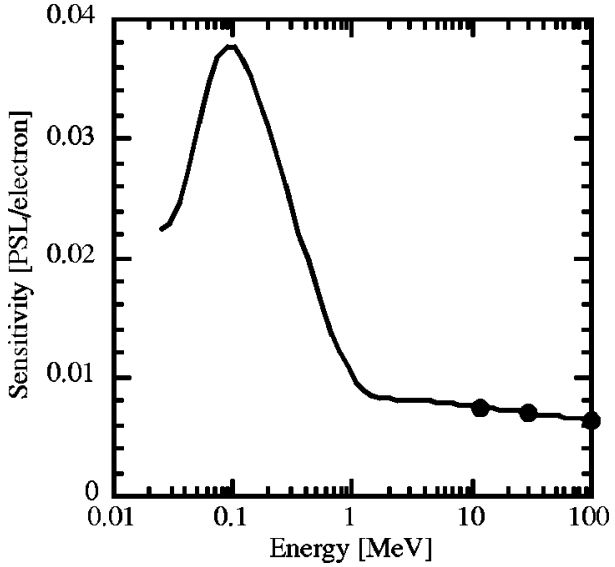

FIG. 7. Calibrated sensitivity curve for electrons up to $100 \mathrm{MeV}$.

LINAC is operated at a multibunch mode and a pulse output is $0.05 \mathrm{nC}$ while the S-band is operated, same as the L-band at the single pulse output of $2.5 \mathrm{nC}$. The electron charge is measured with a Rogovsky coil. IP is placed at $50 \mathrm{~cm}$ away from the output window for the L-band and at $150 \mathrm{~cm}$ for the S-band as shown in Fig. 5. A 6-mm-thick Al diffuser is placed to avoid IP saturation for the case of S-band. The contribution of Bremsstrahlung $\mathrm{x}$ rays is estimated with Monte Carlo simulations using the code EGS4 $4^{11,12}$ and the sensitivity for $\gamma$ rays calibrated using a $\gamma$-ray source. The sensitivity for $\gamma$ rays is one order magnitude smaller than that for electrons.

We define the sensitivity of the IP as PSL intensity per electron. First we make the relative sensitivity curve of electron energy up to $1.25 \mathrm{MeV}^{13}$ absolute by using a $\beta$-ray source ${ }^{147} \mathrm{Pm}$. The maximum energy of electrons emitted from ${ }^{147} \mathrm{Pm}$ is $230 \mathrm{keV}$ and the decay rate is 2.6 years for the half intensity. Irradiation time is $30 \mathrm{~s}$ on the IP and the data are read after $80 \mathrm{~min}$. The sensitivity curve obtained in Ref. 13 is the one with $3 \mu \mathrm{m}$ protective plastic and $110 \mu \mathrm{m}$ phosphor layers in the IP. We have estimated the error caused by using the IP:BAS-SR2025 to be within $8 \%$ from 0.2 to $1.2 \mathrm{MeV}$ with Monte Carlo simulations EGS4. In this estimation, the deposited energies in the phosphor layer of IP are calculated and compared between the situation of the references and our cases.

Figure 6 shows the normalized sensitivity curve of Ref. 13 and the energy spectrum of the ${ }^{147} \mathrm{Pm}$. If the electrons irradiated per second is $f(\varepsilon)$ at an energy $\varepsilon$, we have $\tau$ times $f(\varepsilon)$ electrons in our case at $\varepsilon$ for $\tau$ second irradiation. If the

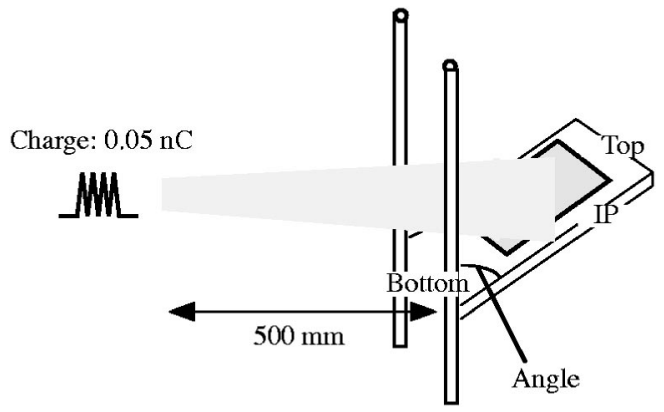

FIG. 8. The layout of the oblique incidence experiments. 

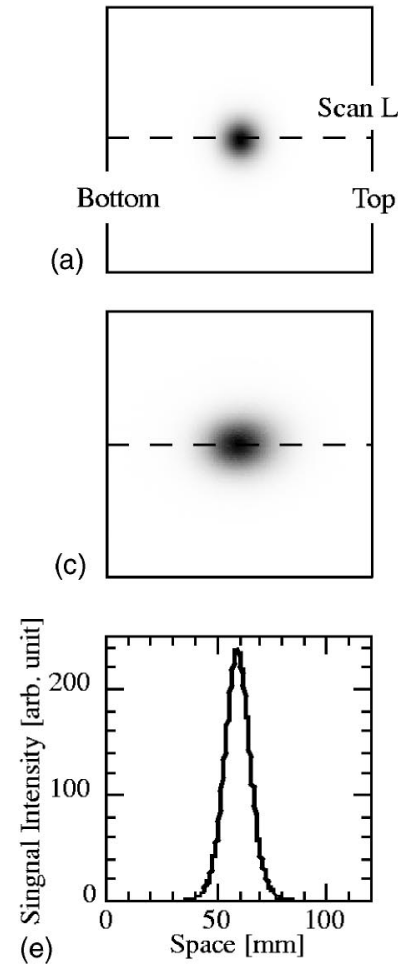

(d)

(b)
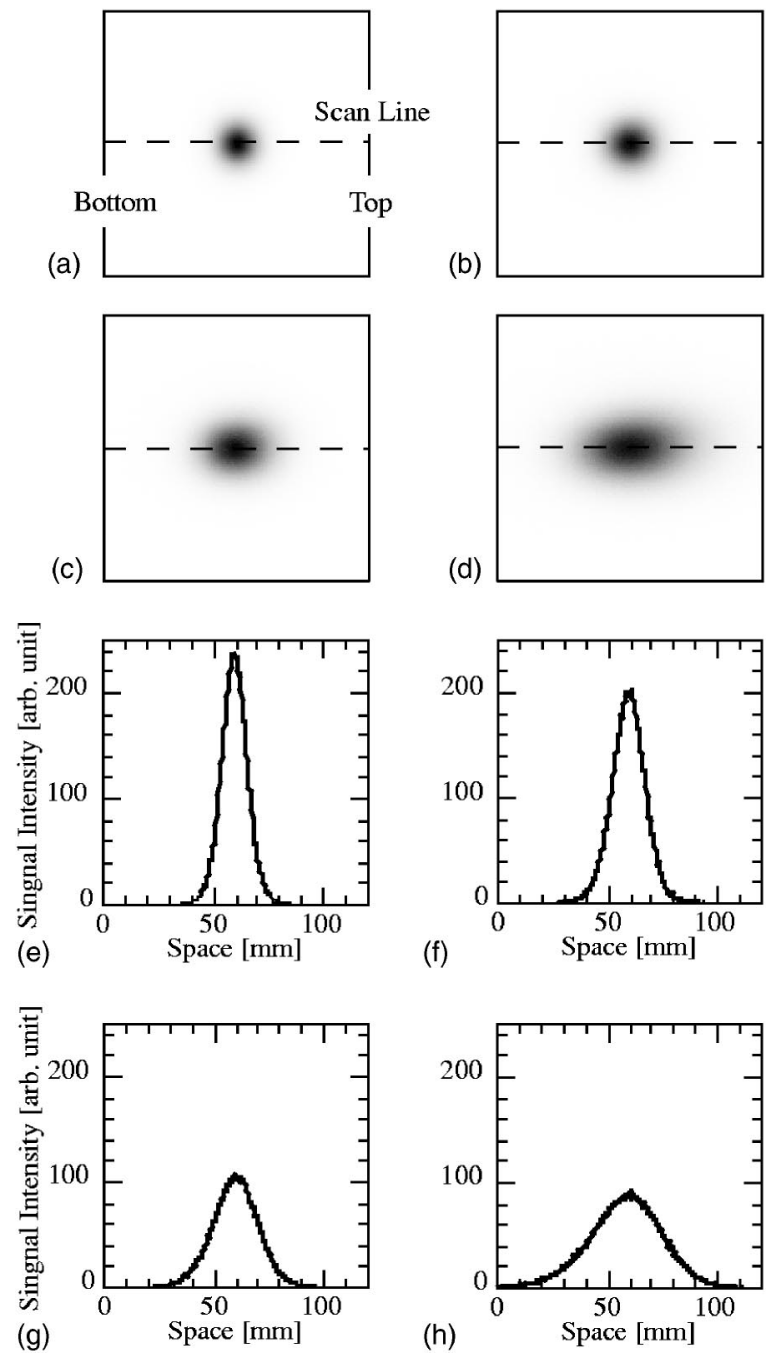

(f)

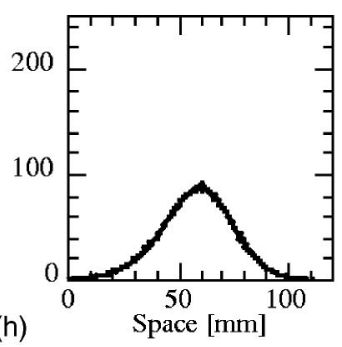

FIG. 9. Electron profile data and line scan of $30 \mathrm{MeV}$ electrons for oblique incidence at (a) $0^{\circ}$, (b) $30^{\circ}$, (c) $45^{\circ}$, (d) $60^{\circ}$.

sensitivity of the IP to the electron is $g(\varepsilon)$ [PSL/electron], then the IP response $q(\varepsilon)$ is given by

$$
q(\varepsilon)=\tau \times f(\varepsilon) \times g(\varepsilon) .
$$

When the $\beta$-ray source energy has some spread, then the IP response in total $\left(Q_{\mathrm{PSL}}\right)$ is given by

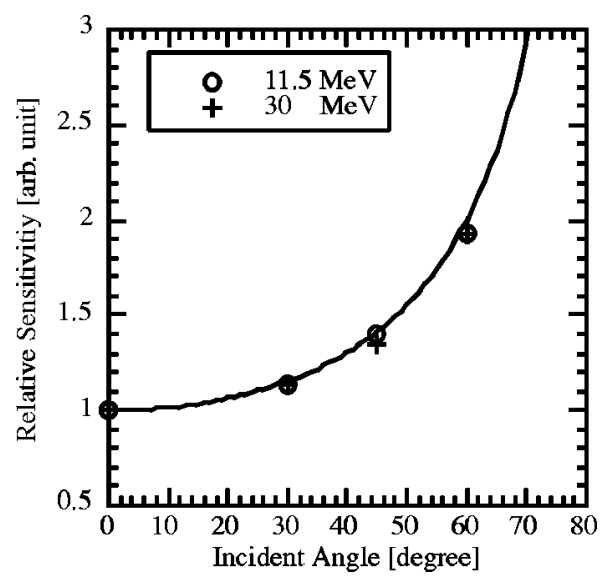

FIG. 10. Relative sensitivity for the oblique incidence. The solid curve is $1 / \cos \theta$ and shows the good fit with the experimental results.

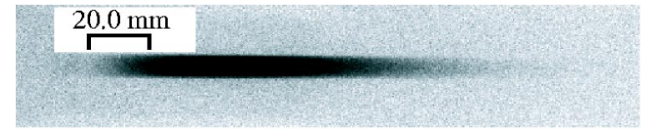

FIG. 11. Raw data of electron spectrum on IP.

$$
Q_{\mathrm{PSL}}=\int q(\varepsilon) d \varepsilon
$$

We introduce the coefficient $\alpha$ to make the absolute sensitivity curve as

$$
g(\varepsilon)=\alpha g_{\mathrm{r}}(\varepsilon),
$$

where $g_{\mathrm{r}}(\varepsilon)$ is the relative sensitivity curve given in Ref. 13 .

We have Eq. (6) using Eqs. (3)-(5),

$$
Q_{\mathrm{PSL}}=\alpha \tau \int f(\varepsilon) \times g_{\mathrm{r}}(\varepsilon) d \varepsilon \text {. }
$$

We obtain $Q_{\mathrm{PSL}}$ from our $\beta$-ray irradiation experiment, then the factor $\alpha$ can be fixed and the absolute sensitivity curve up to $1.25 \mathrm{MeV}$ is given from Eq. (5).

The absolute sensitivity is shown in Fig. 7. PSL values are shown per electron as circles in Fig. 7 for 11.5, 30, and $100 \mathrm{MeV}$ electrons. Since the charge of each electron pulse is known as shown in Table I, it is possible to obtain these calibrations. The calibration values are 0.0074 PSL/electron, $0.0070 \mathrm{PSL} / \mathrm{electron}$, and $0.0064 \mathrm{PSL} / \mathrm{electron}$ for $11.5,30$, and $100 \mathrm{MeV}$ electrons. The main source of the error is due to the error in the electron energy, which are $\pm 5 \%, \pm 5 \%$, and $\pm 10 \%$ for $11.5,30$, and $100 \mathrm{MeV}$ electrons.

From Fig. 7, one can see that the sensitivity is rather constant for the energy range above $1 \mathrm{MeV}$. This sensitivity is consistent with the fact that there is no substantial differ-
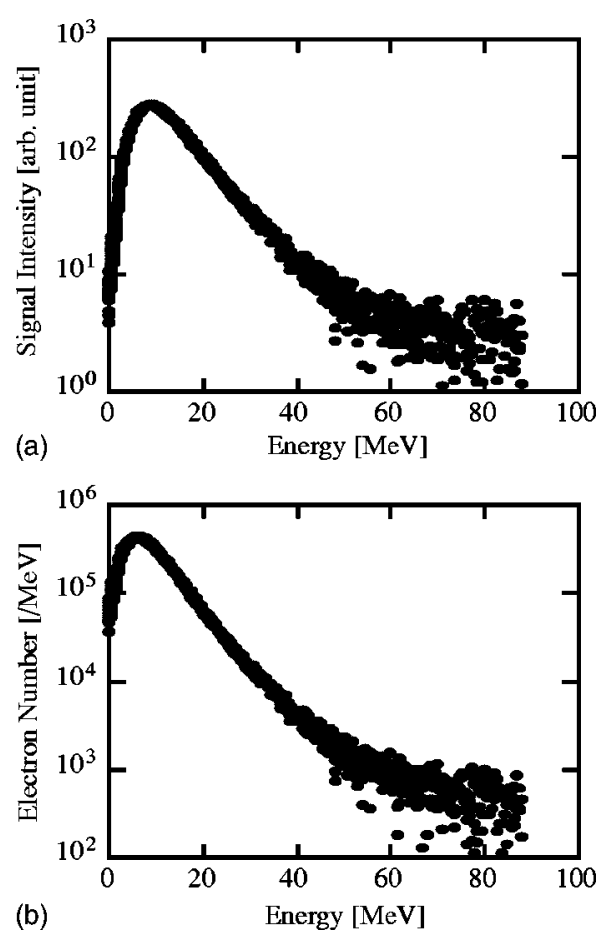

FIG. 12. Signal profile measured by the ESM. (a) Intensity profile of raw data. (b) Electron energy spectrum. 
ence in absorption in the phosphor layer of IP for electron energy above 1 to $100 \mathrm{MeV}$ with the EGS4 code simulation.

\section{Oblique incidence experiment}

We have examined the effect of electrons obliquely incident on the IP at from $0^{\circ}$ to $80^{\circ}$. This situation corresponds to a case where hot electrons are bent with a pair magnet and are incident obliquely on the IP in an electron spectrometer.

This experiment uses electron pulses from the LINAC machine the same as the one used for the calibration experiment. The IP was inclined to the electron pulses. The angles were $0^{\circ}, 30^{\circ}, 45^{\circ}$, and $60^{\circ}$. The schematic is shown in Fig. 8 . Electrons at 11.5 and $30 \mathrm{MeV}$ energies were used from the L-band LINAC. The charge value is $0.05 \mathrm{nC}$ for a single pulse. The IP was placed $50 \mathrm{~cm}$ away from the vacuum window.

The data for each angle are shown in Figs. 9(a)-9(d) which are raw data and (e)-(h) are the profiles at $30 \mathrm{MeV}$. Here the pixel size is $200 \mu \mathrm{m}$. The right-hand side of the profile corresponds to the upper side of the IP. The PSL values drop and are distributed in a wider region with the oblique angle as shown in the line scans (e)-(h). The PSL intensity is shown for the oblique incidence normalized at $0^{\circ}$ in Fig. 10. The sensitivity increases with the oblique angle $(\theta)$ and is fitted very well with $1 / \cos \theta$, which corresponds exactly to the increased path length with the oblique incidence effect.

\section{ELECTRON SPECTRUM MEASURED BY ESM}

We show here an example of hot electron spectrum measured by this electron spectrometer. Figure 11 shows a raw data of electron spectrum recorded on an IP. The electron data were taken at a laser intensity $10^{19} \mathrm{~W} / \mathrm{cm}^{2}$ with a $350 \mathrm{~J}$ laser energy for a 1 ps laser pulse. The target was a gold plane target with a $1 \mathrm{~mm}$ thickness. The target was set at $26^{\circ}$ to the laser axis with a $P$ polarization. The ESM was placed at $37^{\circ}$ in the horizontal plane and $18^{\circ}$ in the vertical plane in the forward direction of the laser axis. Figure 12(a) shows raw signal intensity versus electron energy before the calibration curve is taken care of.

The data reduction is conducted as follows. When we take account of the oblique incidence effect with the oblique angle $\theta$, the IP reading signal intensity $q(\varepsilon)$ is normalized as

$$
I(\varepsilon)=q(\varepsilon) \times \cos \theta,
$$

where $\theta$ also depends on the energy of electrons. The sensitivity $g(\varepsilon)$ shown in Fig. 7 is given for the normal incidence. Hence the electron number $N(\varepsilon)$ is obtained by dividing the $I(\varepsilon)$ by the sensitivity $g(\varepsilon)$, including the fading effect at 80 min after the shot as

$$
N(\varepsilon)=\frac{I(\varepsilon)}{g(\varepsilon) f(80)} .
$$

With all the calibration included, the electron spectrum can be obtained as shown in Fig. 12(b). Figure 12(b) shows the spectrum extending up to $80 \mathrm{MeV}$ and the slope temperature $6 \mathrm{MeV}$.

\section{ADVANTAGES USING IP FOR ESM}

The response linearity of IP for the number of incidence electrons has already been studied ${ }^{10}$ and the linearity is much better than other detectors, such as $\mathrm{x}$-ray film. The linearity enables one to obtain the accurate number of hot electrons using calibrated IP as a detector.

It is shown in this study that IPs have enough high sensitivity to obtain high energy electrons with intense noise generated by laser-plasma interactions. The IP can detect $10^{3}$ electrons which entered one pixel with the energy at over $80 \mathrm{MeV}$.

The spatial resolution of IP can be set smaller than $200 \mu \mathrm{m}$. Actually, the minimum size reading out with BAS1800 is $50 \mu \mathrm{m}$, which is corresponding to the energy resolution smaller than a few kilo-electron-volts. The energy resolution in the spectrometer mainly depends on the spatial resolution of detectors. The spatial resolution is much inferior in cases in which arrayed scintillators are used as a detector in the spectrometer. The good energy resolution is also permitted by using IP in our ESM. If the ratio of signal to background noise level is high enough, the pixel size can be set much smaller and better energy resolution is allowed.

\section{ACKNOWLEDGMENTS}

The authors are thankful for discussions with Dr. J. Zheng, Dr. T. Matsuoka, Dr. L. An-le, T. Kurahashi, M. Tampo, and Professor Ravindra Kumar. T. Yamamoto and S. Suemine at the LINAC system are acknowledged for providing technical support.

${ }^{1}$ J. Miyahara et al., Nucl. Instrum. Methods Phys. Res. A 246, 572 (1986).

${ }^{2}$ Y. Amemiya and J. Miyahara, Nature (London) 336, 89 (1988).

${ }^{3}$ Y. Iwabuchi et al., Jpn. J. Appl. Phys., Part 1 33, 1A (1994).

${ }^{4}$ S. Okano and J. Miyahara, Fujifilm Research and Development 36, 39 (1991).

${ }^{5}$ F. Brunel, Phys. Rev. Lett. 59, 52 (1987); Phys. Fluids 31, 2714 (1988).

${ }^{6}$ W. L. Kruer and K. Estabrook, Phys. Fluids 28, 430 (1985).

${ }^{7}$ K. A. Tanaka et al., Phys. Plasmas 7, 2014 (2000).

${ }^{8}$ R. Kodama et al., Nature (London) 412, 798 (2001).

${ }^{9}$ H. Ohuchi, Jpn. J. Health Phys. 34, 52 (1999) (in Japanese).

${ }^{10}$ A. Taniyama et al., J. Electron Microsc. 45, 232 (1996).

${ }^{11}$ W. R. Nelson, H. Hirayama, and D. W. O. Rogers, "THE EGS4 CODE SYSTEM" (Stanford Linear Accelerator Center, Stanford University, 1985).

${ }^{12} \mathrm{H}$. Hirayama and Y. Namito, Lecture Notes of EGS4 Course at KEK (KEK, Ibaraki, 1999).

${ }^{13}$ A. Taniyama et al., J. Electron Microsc. 46, 303 (1997). 